\title{
Clinical outcomes after percutaneous transforaminal endoscopic discectomy for lumbar disc herniation: a prospective case series
}

\author{
Pravesh S. Gadjradj, BSc, ${ }^{1-3}$ Maurits W. van Tulder, PhD, ${ }^{3}$ Clemens M. F. Dirven, MD, PhD, ${ }^{1}$ \\ Wilco C. Peul, MD, PhD, MBA, ${ }^{2}$ and B. Sanjay Harhangi, MD, PhD ${ }^{1,4}$ \\ ${ }^{1}$ Department of Neurosurgery, Erasmus Medical Center; ${ }^{4}$ Department of Neurosurgery, Park Medical Center, Rotterdam; \\ 2Department of Neurosurgery, Leiden University Medical Center, Leiden; ${ }^{3}$ Faculty of Earth and Life Sciences, Department of \\ Health Sciences, VU University Amsterdam, The Netherlands
}

\begin{abstract}
OBJECTIVE Throughout the last decades, full-endoscopic techniques to treat lumbar disc herniation (LDH) have gained popularity in clinical practice. To date, however, no Class I evidence on the efficacy of percutaneous transforaminal endoscopic discectomy (PTED) has been published, and studies describing its safety and short- and long-term efficacy are scarce. In this study the authors aimed to evaluate the clinical outcomes and safety in patients undergoing PTED for LDH.

METHODS Patients who underwent PTED for LDH between January 2009 and December 2012 were prospectively followed. The primary outcomes were the visual analog scale (VAS) score for leg pain and the score on the Quebec Back Pain Disability Scale (QBPDS). Secondary outcomes were the perceived experience with the local anesthesia used and satisfaction with the results after 1 year using Likert-type scales. The pretreatment means were compared with the means obtained 6 and 52 weeks after surgery using paired t-tests.
\end{abstract}

RESULTS A total of 166 patients underwent surgery for a total of $167 \mathrm{LDHs}$. The mean duration of surgery $( \pm$ SD) waS $51.0 \pm 9.0$ minutes. The 1-year follow-up rate was $95.2 \%$. The mean reported scores on the VAS and QBPDS were 82.5 $\pm 17.3 \mathrm{~mm}$ and $60.0 \pm 18.4$ at baseline, respectively. Six weeks after surgery, the scores on the VAS and QBPDS were significantly reduced to $28.8 \pm 24.5 \mathrm{~mm}$ and $26.7 \pm 20.6$, respectively $(p<0.001)$. After 52 weeks of follow-up, the scores were further reduced compared with baseline scores $(p<0.001)$ to $19.6 \pm 23.5 \mathrm{~mm}$ on the VAS and $20.2 \pm 18.1$ on the QBPDS. A total of 4 complications were observed, namely 1 dural tear, 1 deficit of ankle dorsiflexion, and 2 cases of transient paresis in the foot due to the use of local anesthetics.

CONCLUSIONS PTED appears to be a safe and effective intervention for LDH and has similar clinical outcomes compared to conventional open microdiscectomy. High-quality randomized controlled trials are required to study the efficacy and cost-effectiveness of PTED.

http://thejns.org/doi/abs/10.3171/2015.10.FOCUS15484

KEY WORDS lumbar disc herniation; percutaneous transforaminal endoscopic discectomy; minimally invasive spine surgery

$\mathrm{W}$ ITH an annual incidence of 5 cases per 1000 persons, the lumbosacral radicular syndrome caused by a lumbar disc herniation (LDH) is a frequently observed problem. ${ }^{14}$ The most important symptom is lumbosacral radicular leg pain following a dermatomal pattern from below the knee till the feet and toes. The pain worsens with coughing. Other clinical findings may include unilateral spasm of the paraspinal muscles, gait deformity, limited forward flexion, and sensory deficits such as muscle weakness and reflex changes. ${ }^{20,27}$

Symptomatic LDH can be managed variably: conservatively, with interventional pain treatment, or surgically. ${ }^{11}$ In patients with persistent or progressive symptoms after 6 to 12 weeks of conservative treatment (by analgesics or by

ABBREVIATIONS LDH = lumbar disc herniation; PTED = percutaneous transforaminal endoscopic discectomy; QBPDS = Quebec Back Pain Disability Scale; SAP = superior articular process; VAS = visual analog scale. 
reducing mechanical pressure on the nerve root-e.g., by physical therapy), surgery is indicated. A previous study showed that patients who were randomized to undergo early surgery had disability scores after 1 year that were similar to those of patients who underwent prolonged conservative management (assigned to conservative treatment with eventual surgery if needed).$^{19}$ However, patients in the early surgery group reported earlier relief of leg pain and reported a faster rate of recovery, demonstrating the benefits of surgery.

In the beginning of the previous century, Mixter and Barr performed the first lumbar disc surgery. ${ }^{17}$ Since then several surgical techniques have been described for the treatment of the $\mathrm{LDH}$, including minimally invasive approaches to access the lumbar disc, ${ }^{13}$ which were developed with the aim of limiting the skin incision and decreasing muscle damage and epidural scarring. Several lateral percutaneous techniques have been described since. The indications for these percutaneous procedures were mostly limited to contained LDHs.

Currently, open microdiscectomy is still the gold standard procedure for treating $\mathrm{LDH},{ }^{2}$ albeit without Class I evidence. Improvements in the use and design of optics and surgical instruments have led to the utilization of fullendoscopic surgical procedures, such as the percutaneous endoscopic lumbar discectomy. The surgical efficacy of these endoscopic techniques is expected to be at least comparable to that of the conventional, more invasive open procedures, but with reduced hospitalization and a shorter time to recover. Percutaneous transforaminal endoscopic discectomy (PTED) is a minimally invasive treatment in which the incision size is further reduced, to approximately $8 \mathrm{~mm}$. Furthermore, no paraspinal muscle is cut or detached from the insertion. Consequently, PTED is thought to have a further reduced invasiveness as well as result in reduced muscle and epidural scarring. Since its establishment, PTED has been shown to be a promising minimally invasive technique. ${ }^{23}$ The minimal tissue damage during PTED could make a difference in effectiveness, and this could potentially lead to a lower intensity of both leg and back pain, faster rehabilitation and integration, and thus lower costs for society. However, studies describing the safety and short- and long-term efficacy of PTED are scarce. In this article we describe our experience with a large series of patients who underwent PTED for LDH.

\section{Methods}

All patients presenting with sciatica were primarily evaluated by neurologists. If the symptoms persisted, MRI of the lumbar spine was requested. Patients who were diagnosed with LDH and had persisting signs and symptoms were referred to Park Medical Center and examined by the neurosurgeon (B.S.H.). When surgery was indicated written informed consent was obtained from all patients to undergo PTED within 2 weeks after consultation. This study was approved by the institutional review board of Park Medical Center and the medical ethics committee of Erasmus Medical Center.

\section{Patient Population}

Patients between the ages of 18 and 80 years who pre- sented for surgical treatment of LDH between 2009 and 2012 were enrolled in this study. The indication for surgery was according to Dutch guidelines, which included an MRI study showing an LDH with or without concomitant spinal or lateral recess stenosis or sequestration in patients with at least 16 weeks of persistent radicular irritation with or without motor or sensory loss. Furthermore, for patients with at least 6 weeks of excessive radiating leg pain with no tendency for any clinical improvement surgery was also indicated. ${ }^{8}$ PTED was only contraindicated in patients with severe isthmic spondylolisthesis or severe congenital spinal canal stenosis. Patients who had undergone previous surgery on the same spinal level, were pregnant, were diagnosed with the cauda equine syndrome, or had inadequate knowledge of the Dutch language were excluded from the study.

\section{Surgical Procedure}

All procedures were performed under local anesthesia and sedation with propofol and remifentanil. Patients were placed in a prone position on a Wilson frame. The surgeon operated from the side of the disc prolapse, and the video monitor and $\mathrm{C}$-arm were positioned on the opposite side. After the position of the iliac crest was determined, ${ }^{21,22}$ the skin incision was marked $12 \mathrm{~cm}$ from the midline at L5-S1, $10 \mathrm{~cm}$ from the midline at L4-5, and $8 \mathrm{~cm}$ from the midline at L3-4 and L2-3. Following disinfection and sterile draping, the entry point was marked, always superior to the iliac crest, and a line was drawn across the superior articular process (SAP) to the midline of the lower endplate (Fig. 1). Then the skin was infiltrated with $2-3 \mathrm{ml}$ lidocaine $1 \%$, and an 8-mm skin incision was made. An 18 -gauge needle was introduced to the SAP of the lower vertebra of the disc herniation using anteroposterior and lateral fluoroscopy (Fig. 2). The SAP was infiltrated with $2-3 \mathrm{cc}$ of lidocaine $1 \%$. A guidewire was introduced via the 18-gauge needle, and the needle was then removed. Next, 2 conical rods were introduced over the guidewire to stretch the soft tissue. After dilation, a 4-mm drill and then, successively, 6-mm and 8-mm drills were used to enlarge the safe caudal part of the neuroforamen, also known as Kambin's triangle, ${ }^{12}$ while keeping the guidewire in place. Sometimes, a 9-mm drill was used to further enlarge the neuroforamen. The working cannula was introduced over the second dilatator until the tip was located on the disc herniation, close to the posterior longitudinal ligament. The endoscope with working channel and irrigation channel was introduced, and the herniated disc material was removed in one part or piecemeal with a disc forceps (Fig. 3). The decompression was considered sufficient if the nerve showed pulsations similar to that of the heart rate and the amount of removed disc material matched the amount seen on the MRI. The skin was closed with an intracutaneously dissolving stitch. The blood loss associated with this procedure was minimal (less than 1-5 ml), although no objective measurements could be performed.

\section{Outcomes}

Primary outcomes of this study were the scores on the Quebec Back Pain Disability Scale (QBPDS) ${ }^{24}$ and a vi- 


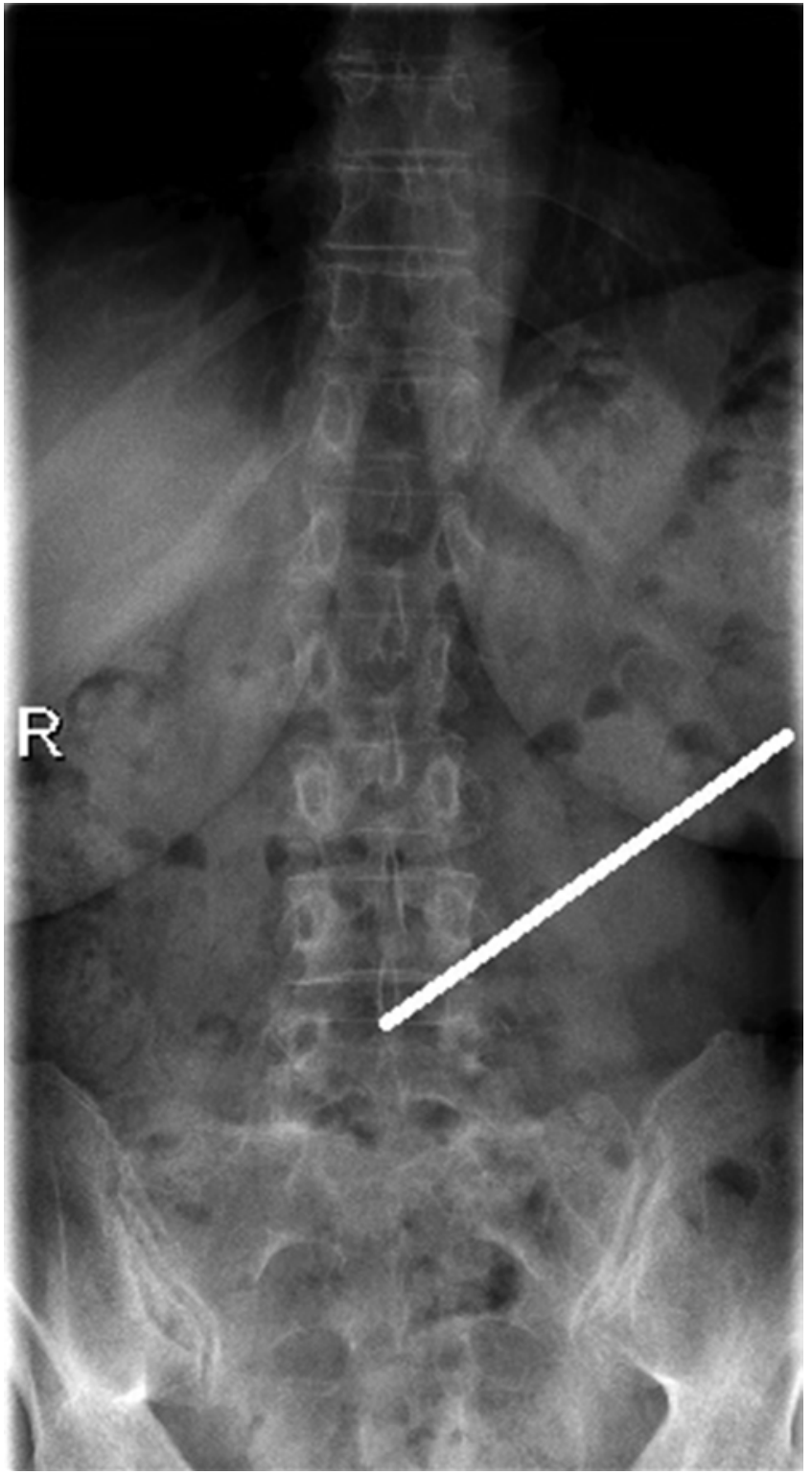

FIG. 1. Fluoroscopic image showing the line drawn across the SAP to the midline of the lower endplate.

sual analog scale (VAS) ${ }^{6}$ for leg pain. The QBPDS measures the disabilities caused by back pain, ranging from 0 for no functional impairment to 100 indicating maximum functional impairment. The VAS for leg pain measures the pain from $0 \mathrm{~mm}$, indicating no pain, to $100 \mathrm{~mm}$ representing the worst pain ever experienced in the leg. The QBPDS and the VAS scores were assessed at baseline and 6 and 52 weeks prospectively. Patients were followed for 6 weeks after surgery at the outpatient clinic and discharged if no signs of radiculopathy were present. In case of recurrent or persistent radiculopathy, a postoperative MRI was requested to identify recurrence of $\mathrm{LDH}$.

Furthermore, patients were asked to indicate their experience with local anesthetics on a 5-point Likert-type scale $^{1}$ postoperatively, with a score of 0 indicating a very bad experience and a score of 5 a very good experience. After 1 year of follow-up, the patients' satisfaction with
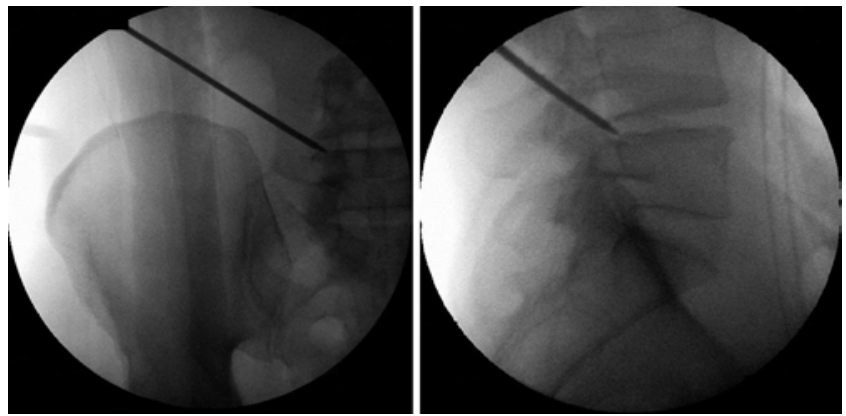

FIG. 2. Anteroposterior (left) and lateral (right) intraoperative fluoroscopic images of the guidewire placed at level L4-5 where the disc herniation is present.

the result of PTED was measured using 2 questions. Patients were asked whether they would undergo the surgery again if they would experience the same complaints and whether they would recommend the PTED technique to other patients with similar cases. Any new symptoms, complications of surgery, or recurrence of LDH were also evaluated after 52 weeks of follow-up.

\section{Statistical Analyses}

Descriptive statistics were used to analyze demographic data and Likert-type scales. Paired t-tests were performed to compare pre- and postoperative scores on the QBPDS and VAS. Results are presented as means with standard deviations. A p value less than 0.05 was considered statistically significant. All statistical analyses were performed with SPSS (version 21.0, IBM Corp.).

\section{Results}

From January 2009 through December 2012, 166 patients underwent PTED for treatment of 167 LDHs. Conversion to open microdiscectomy was not required in any of these cases. At 52 weeks of follow-up, data were available for 158 patients $(95.2 \%)$. The remaining 8 patients were lost to follow-up for the following reasons: relocating without leaving a forwarding address (6 patients), being out of the country for an extended period (1 patient), and death due to a cause unrelated to the surgery (1 patient). The mean age of our study population was $43.5 \pm 13.5$ years. The most common level of LDH was L5-S1 (49.1\%), followed by L4-5 (41.3\%), L3-4 (8.4\%), and L2-3 (1.2\%). At baseline the mean reported score on the QBPDS was 60.0 (range 18-100). The mean VAS score for leg pain was $82.5 \mathrm{~mm}$ (range 5-100 $\mathrm{mm}$ ) (Table 1).

The duration of surgery varied from 34 to 94 minutes (mean 51.0 minutes). Thirty-three patients (20.8\%) reported a neutral experience with the local anesthetics, while $102(64.2 \%)$ patients had a good or very good experience (Table 2). Complications occurred in 4 cases (2.4\%): 1 dural tear occurred, 1 patient experienced dysfunction in the dorsiflexion of the foot ankle, and 2 patients had a transient paralysis due to lidocaine anesthetics in the neuroforamen. No postoperative wound infections, thrombosis, or hemorrhages were observed; 164 (98.8\%) of the patients could be discharged successfully 2 hours after surgery, and the remaining 2 patients (1.2\%) could be discharged 1 


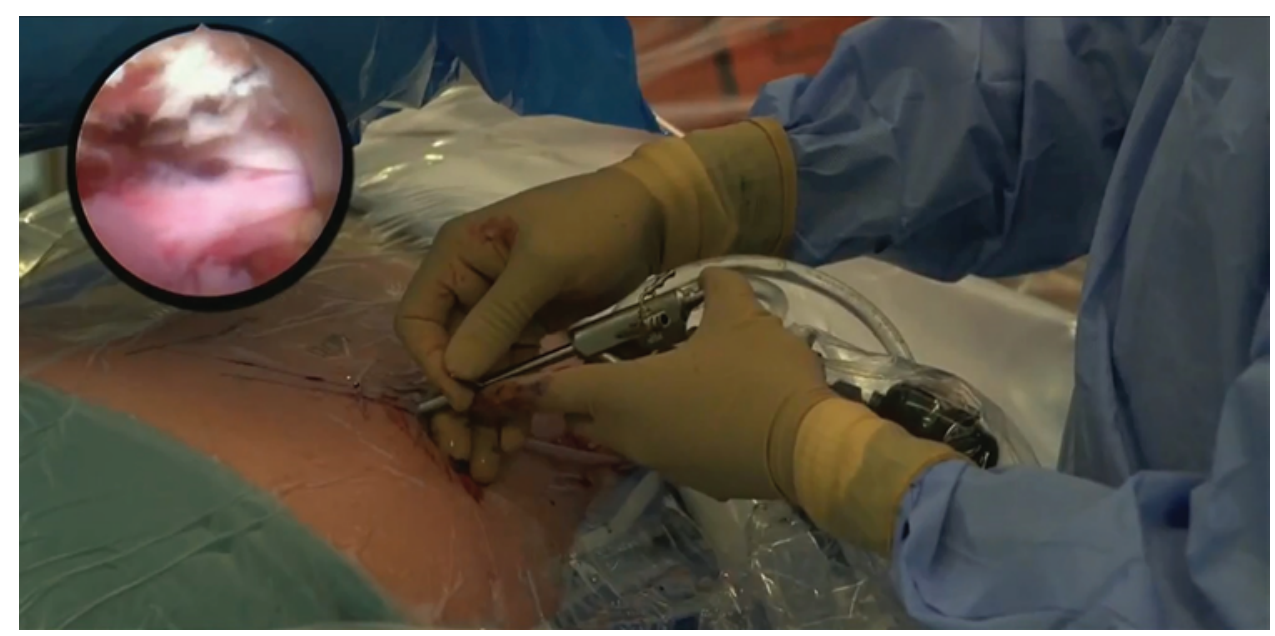

FIG. 3. Intraoperative photograph. With the working channel placed at the right level, it is possible to remove the herniated disc material with the rongeur. The inset in the left upper corner shows the view through the endoscope.

day after surgery. Twelve patients (7.2\%) needed to undergo additional surgery within 1 year after PTED; 11 (6.6\%) of these patients had a recurrence of the disc herniation, and 1 patient developed a de novo LDH at another level.

Figure 4 shows the course of our primary outcome parameters at baseline and 6 and 52 weeks after surgery. After 6 weeks of follow-up, the mean reported score on the QPBDS decreased significantly $(\mathrm{p}<0.001)$, with a mean difference of 33.3 points (95\% CI 29.5-36.4), and the mean VAS for leg pain decreased by a mean of 53.7 $\mathrm{mm}(95 \%$ CI $49.4-58.3 \mathrm{~mm})(\mathrm{p}<0.001)$ (Table 3). Fiftytwo weeks after surgery, the mean score on the QBDS and VAS leg decreased further to 20.2 and $19.6 \mathrm{~mm}$ ( $p<0.001$ for both, in comparison with baseline scores, although the differences were not statistically significant in comparison with the 6-week follow-up scores).

Finally, 154 patients $(92.8 \%)$ indicate that they would

TABLE 1. Baseline characteristics of the 166 patients in this study*

\begin{tabular}{lc}
\hline \multicolumn{1}{c}{ Characteristic } & Value \\
\hline Sex & \\
\hline Male & $85(51.2 \%)$ \\
\hline Female & $81(48.8 \%)$ \\
\hline Mean age $(\mathrm{yrs})$ & $43.5 \pm 13.5$ \\
\hline Mean body mass index $\left(\mathrm{kg} / \mathrm{m}^{2}\right)$ & $27.1 \pm 5.0$ \\
\hline Mean duration of symptoms $(\mathrm{wks})$ & $51.2 \pm 15.3$ \\
\hline Level of disc herniationt & \\
\hline L2-3 & $2(1.2 \%)$ \\
\hline L3-4 & $14(8.4 \%)$ \\
\hline L4-5 & $69(41.3 \%)$ \\
\hline L5-S1 & $82(49.1 \%)$ \\
\hline Mean QBPDS & $60.0 \pm 18.4$ \\
\hline Mean VAS $(\mathrm{mm})$ & $82.5 \pm 17.3$ \\
\hline
\end{tabular}

* Values represent numbers of patients unless otherwise indicated. Mean values are presented with SDs. For a description of QBPDS and VAS scoring, please refer to the Outcomes section of Methods.

$\dagger$ One patient underwent PTED at both L4-5 and L5-S1. recommend the PTED technique to people with similar complaints caused by LDH. When asked whether they would undergo the PTED again, if they knew that they would experience the same complaints due to recurrent LDH, 152 patients (91.6\%) answered positively.

\section{Discussion}

The results of this study showed statistically significant relief of back disabilities and leg pain in the short term and improvement on these results over the long term. The mean duration of surgery was less than 1 hour, and the recurrence rate for repeated LDH surgery was $6.6 \%$ within 1 year in this group with a high follow-up percentage (95.6\%). Furthermore, almost all (98.8\%) of the patients

TABLE 2. Operative characteristics of the patient population

\begin{tabular}{cc}
\hline \multicolumn{1}{c}{ Characteristic } & Value \\
\hline Mean duration of surgery (mins) & $51.0 \pm 9.0$ \\
\hline Complications & $4(2.4 \%)$ \\
\hline Dural tear & $1(0.6 \%)$ \\
\hline Ankle/toe dorsiflexion weakness & $1(0.6 \%)$ \\
\hline Transient paresis & $2(1.2 \%)$ \\
\hline Day of mobilization & $164(98.8 \%)$ \\
\hline Day of surgery & $2(1.2 \%)$ \\
\hline Day 1 after surgery & $12(7.2 \%)$ \\
\hline Repeated surgery w/in 1 yr & $11(6.6 \%)$ \\
\hline Recurrent LDH at same level & $1(0.6 \%)$ \\
\hline LDH at another level & $159(95.8 \%)$ \\
\hline Experience w/ local anesthesia* & $2(1.2 \%)$ \\
\hline Very bad & $3(1.9 \%)$ \\
\hline Bad & $33(20.8 \%)$ \\
\hline Neutral & $51(32.1 \%)$ \\
\hline Good & $51(32.1 \%)$ \\
\hline Very good
\end{tabular}

* Experience with local anesthesia was assessed using the 5-point Likert-type scale. A score of 0 corresponds with a very bad experience, while a score of 5 corresponds with a very good experience. 


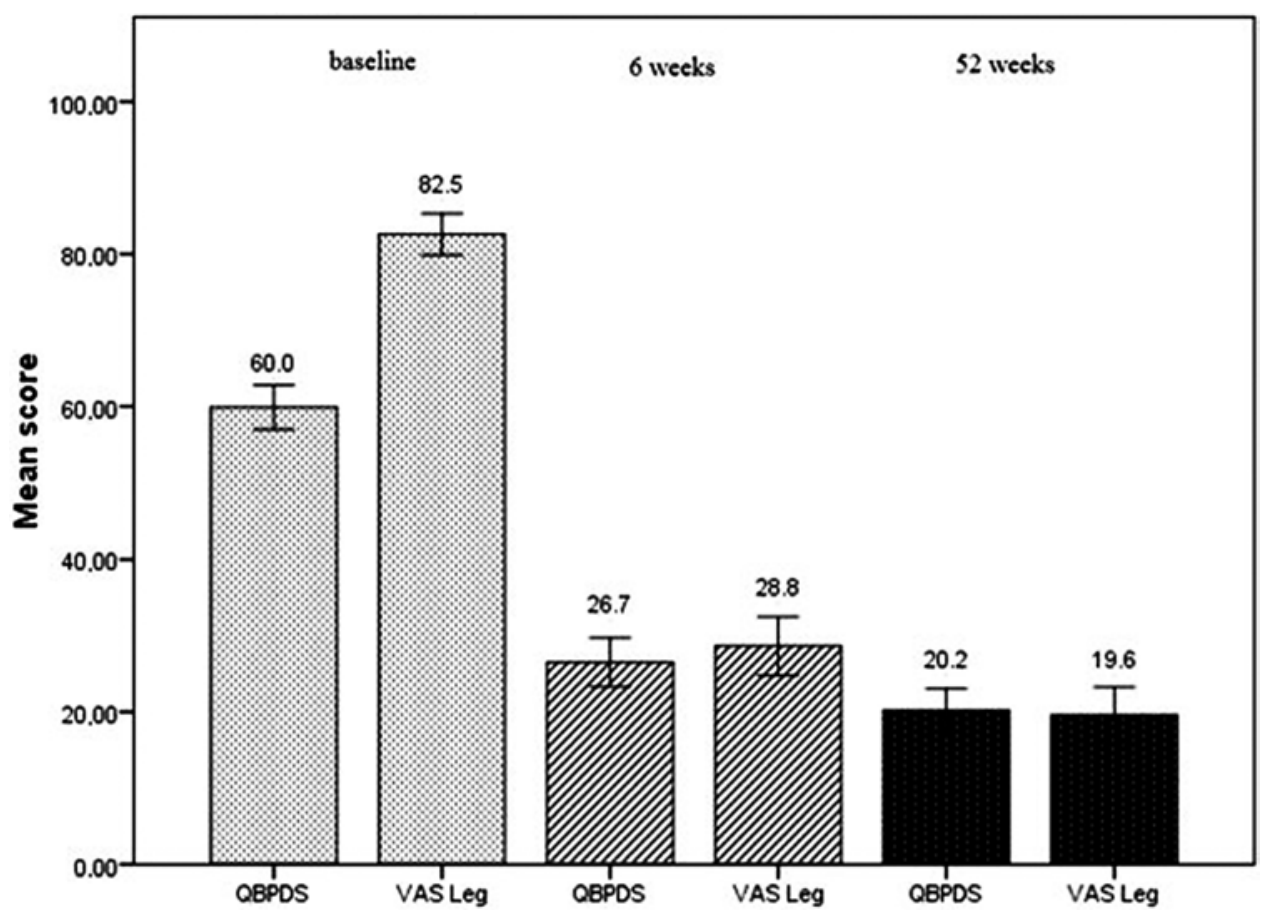

FIG. 4. Graph showing the mean values of the primary outcome measures for 166 patients at baseline and after 6 and 52 weeks of follow-up. For details on the QBPDS and VAS scoring, please refer to the Outcomes section of Methods.

could be discharged on the day of surgery. Only $2.4 \%$ of the 166 patients experienced minor complications. Finally, as we observed no need for conversion to open microdiscectomy, we can see PTED as equipollent in its potential to treat $\mathrm{LDH}$.

Studies of PTED for LDH describing its safety and short- and long-term efficacy are scarce. However, some other research groups have also evaluated results of the PTED technique ${ }^{13,18}$ and have demonstrated comparable efficacy. In a large single-center retrospective review of 10,228 cases, one research group investigated why PTED failed in treating $\mathrm{LDH}^{5}$ and found that failure was mostly explained by incomplete removal of herniated disc material. Furthermore, they found a short-term recurrence rate of $4.3 \%$, which is comparable to our data. Other studies report similar recurrence rates. ${ }^{4,25,29}$ Another research group published the short-term clinical results of 163 patients who underwent either PTED or full-endoscopic interlaminar discectomy. ${ }^{25}$ They reported a recurrent LDH rate of
$2.8 \%$ in the PTED group, which is lower than our rate. An important reason for this difference could be that the patients in their study were selected to undergo either PTED or interlaminar endoscopic surgery on the basis of CT imaging. Most endoscopic LDH operations at level L5-S1 $(94.2 \%)$ were preferably performed using the interlaminar technique, whereas in our study all patients were operated on using the transforaminal approach. In our case series, all patients with primary LDH were eligible for PTED unless it was contraindicated. The distribution of the level of the disc herniation showed the greatest frequency for L5S1, followed by L4-5, L3-4, and L2-3. This along with a mean age of 43.5 years suggests that our series may be a good reflection of the general Dutch population.

The amount of observed complications, 4 in total, was lower than expected. We did not observe any infection, thrombosis, or hemorrhage. One patient experienced foot drop, while 2 patients experienced transient paralysis. Transient paralysis can be prevented by infiltrating with

TABLE 3. Effects of treatment on primary and secondary outcomes*

\begin{tabular}{lrrrr}
\hline \multicolumn{1}{c}{ Outcome } & Wh & \multicolumn{1}{c}{ Score } & Mean Difference from Baseline $(95 \%$ Cl) & $\mathrm{p} \mathrm{Value}$ \\
\hline Mean QBPDS score & 0 & $60.0 \pm 18.4$ & & \\
\hline & 6 & $26.7 \pm 20.6$ & $33.3(29.5-36.4)$ & $<0.001$ \\
\hline Mean VAS score for leg pain & 52 & $20.2 \pm 18.1$ & $39.8(36.1-43.3)$ & $<0.001$ \\
\hline & 0 & $82.5 \pm 17.3$ & & $<0.001$ \\
\hline & 6 & $28.8 \pm 24.5$ & $53.7(49.4-58.3)$ & $<0.001$ \\
\hline Would recommend in similar cases & 52 & $19.6 \pm 23.5$ & $62.9(58.5-67.5)$ & \\
\hline Would do it again under similar circumstances & 52 & $154(92.8 \%)$ & & \\
\hline
\end{tabular}

\footnotetext{
* Mean values are presented with SDs.
} 
less lidocaine in the neuroforamen. Using too much local anesthetic in the foramen can also lead to a reduction in direct feedback from the patient when the surgeon approaches the nerve root during surgery. Nevertheless, our rate of complications was very low and comparable to the rates reported in the literature. .0,25,26,29 $^{2}$

Nellensteijn et al..$^{18}$ were the first to provide a systematic review of the PTED technique. Their review identified only 1 randomized controlled trial with a low risk of bias. ${ }^{9}$ However, this trial has a disputable generalizability due to inclusion of only certain types of LDHs, namely intracanalicular disc herniations. In a more recently published review and meta-analysis, ${ }^{7}$ Cong et al. pooled results comparing endoscopic discectomy versus open microdiscectomy and found a significantly higher satisfaction rate in patients who underwent endoscopic discectomy. Furthermore, endoscopic surgery was associated with less blood loss and a shorter duration of hospitalization. No significant differences in operating time, recurrence, or complication rates were found. Both reviews, however, emphasize the need for more independent high-quality randomized controlled trials that also assess cost-effectiveness.

Another positive finding was the good or very good experience that the majority of patients had with the local anesthesia. A recently published study reported on the preoperative anxiety that patients experienced before spinal surgery under general anesthesia. ${ }^{16}$ The authors stated that among the 157 patients they included for their study, spinal surgery was the main cause of the anxiety in $74 \%$. General anesthesia was the main cause of anxiety for the other $26 \%$ of the patients awaiting laminectomy or discectomy. Perhaps for these patients, PTED is a better alternative than conventional open microdiscectomy.

An important strength of this study is the prospective collection of data on consecutive patients reducing the possibility of selection and recall bias. Another strength is our limited loss to follow-up. The present study also has several limitations. Due to the design, a proper control group is lacking; however, as previously mentioned, the objective of this study was not to emphasize the merits of PTED over other procedures, but to share the short- and long-term results that show its potential. Moreover, PTED also has a long learning curve due to the concept of a $2 \mathrm{D}$ view. Surgeons are exposed to different landmarks, another direction of approach, and a laborious identification of anatomical structures during surgery. ${ }^{3,15,28}$ Considering the long learning curve of PTED and potential bias, all surgeries were performed by a single neurosurgeon (B.S.H.) who already had extensive experience in performing the PTED technique.

\section{Conclusions}

Based on our findings, PTED seems to be a promising technique to effectively treat LDH. The reported complication rate of PTED is low, as is the percentage of patients requiring additional surgery due to recurrent LDH. Due to its steep learning curve, however, PTED should be further investigated before widespread implementation. Open microdiscectomy remains the current standard therapy for the surgical decompression of LDH. High-quality ran- domized controlled trials are needed to generate Class I evidence on the efficacy and cost-effectiveness of PTED.

\section{Acknowledgments}

We gratefully acknowledge the administrative contribution of M. Smit, S. Moelker, K. Jung, and G. Korst to this study and Ms. Korinne Zwiers for critically evaluating the manuscript.

\section{References}

1. Allen IA, Seaman CA: Likert scales and data analyses. Quality Progress:64-65, 2007

2. Caspar W: A new surgical procedure for lumbar disk herniation causing less tissue damage through a microsurgical approach. Adv Neurosurg 4:74-77, 1977

3. Chaichankul C, Poopitaya S, Tassanawipas W: The effect of learning curve on the results of percutaneous transforaminal endoscopic lumbar discectomy. J Med Assoc Thai 95 (Suppl 10):S206-S212, 2012

4. Choi KC, Kim JS, Ryu KS, Kang BU, Ahn Y, Lee SH: Percutaneous endoscopic lumbar discectomy for L5-S1 disc herniation: transforaminal versus interlaminar approach. Pain Physician 16:547-556, 2013

5. Choi KC, Lee JH, Kim JS, Sabal LA, Lee S, Kim H, et al: Unsuccessful percutaneous endoscopic lumbar discectomy: a single-center experience of 10,228 cases. Neurosurgery 76:372-381, 2015

6. Collins SL, Moore RA, McQuay HJ: The visual analogue pain intensity scale: what is moderate pain in millimetres? Pain 72:95-97, 1997

7. Cong L, Zhu Y, Tu G: A meta-analysis of endoscopic discectomy versus open discectomy for symptomatic lumbar disk herniation. Eur Spine J [epub ahead of print], 2015

8. Dutch Society for Neurology: [Guideline Lumbosacral Radicular Syndrome.] Utrecht: Quality Institute for Health Care CBO, 2008 (Dutch)

9. Hermantin FU, Peters T, Quartararo L, Kambin P: A prospective, randomized study comparing the results of open discectomy with those of video-assisted arthroscopic microdiscectomy. J Bone Joint Surg Am 81:958-965, 1999

10. Hoogland T, van den Brekel-Dijkstra K, Schubert M, Miklitz B: Endoscopic transforaminal discectomy for recurrent lumbar disc herniation: a prospective, cohort evaluation of 262 consecutive cases. Spine (Phila Pa 1976) 33:973-978, 2008

11. Jacobs WC, van Tulder M, Arts M, Rubinstein SM, van Middelkoop M, Ostelo R, et al: Surgery versus conservative management of sciatica due to a lumbar herniated disc: a systematic review. Eur Spine J 20:513-522, 2011

12. Kambin P, Sampson S: Posterolateral percutaneous suctionexcision of herniated lumbar intervertebral discs. Report of interim results. Clin Orthop Relat Res (207):37-43, 1986

13. Kamper SJ, Ostelo RW, Rubinstein SM, Nellensteijn JM, Peul WC, Arts MP, et al: Minimally invasive surgery for lumbar disc herniation: a systematic review and meta-analysis. Eur Spine J 23:1021-1043, 2014

14. Konstantinou K, Dunn KM: Sciatica: review of epidemiological studies and prevalence estimates. Spine (Phila Pa 1976) 33:2464-2472, 2008

15. Lee DY, Lee SH: Learning curve for percutaneous endoscopic lumbar discectomy. Neurol Med Chir (Tokyo) 48:383-389, 2008

16. Lee JS, Park YM, Ha KY, Cho SW, Bak GH, Kim KW: Preoperative anxiety about spinal surgery under general anesthesia. Eur Spine J [epub ahead of print], 2015

17. Mixter WJ, Barr JS: Rupture of the intervertebral disc with involvement of the spinal canal. N Engl J Med 211:210-215, 1934

18. Nellensteijn J, Ostelo R, Bartels R, Peul W, van Royen B, van 
Tulder M: Transforaminal endoscopic surgery for symptomatic lumbar disc herniations: a systematic review of the literature. Eur Spine J 19:181-204, 2010

19. Peul WC, van Houwelingen HC, van den Hout WB, Brand R, Eekhof JA, Tans JT, et al: Surgery versus prolonged conservative treatment for sciatica. N Engl J Med 356:2245-2256, 2007

20. Ropper AH, Zafonte RD: Sciatica. N Engl J Med 372:1240_ 1248,2015

21. Ruetten S, Komp M, Godolias G: An extreme lateral access for the surgery of lumbar disc herniations inside the spinal canal using the full-endoscopic uniportal transforaminal approach-technique and prospective results of 463 patients. Spine (Phila Pa 1976) 30:2570-2578, 2005

22. Ruetten S, Komp M, Merk H, Godolias G: Full-endoscopic interlaminar and transforaminal lumbar discectomy versus conventional microsurgical technique: a prospective, randomized, controlled study. Spine (Phila Pa 1976) 33:931939, 2008

23. Scheckenbach C, Hoogland T: Endoskopische transforaminale Diskektomie (ETD)-Ergebnisse nach 2 Jahren. Orthopadische Praxis 35:104-105, 1999

24. Schoppink LE, van Tulder MW, Koes BW, Beurskens SA, de Bie RA: Reliability and validity of the Dutch adaptation of the Quebec Back Pain Disability Scale. Phys Ther 76:268275, 1996

25. Sencer A, Yorukoglu AG, Akcakaya MO, Aras Y, Aydoseli A, Boyali O, et al: Fully endoscopic interlaminar and transforaminal lumbar discectomy: short-term clinical results of 163 surgically treated patients. World Neurosurg 82:884-890, 2014

26. Türk CC, Kara NN, Biliciler B, Karasoy M: Clinical outcomes and efficacy of transforaminal lumbar endoscopic discectomy. J Neurosci Rural Pract 6:344-348, 2015
27. van Tulder M, Peul W, Koes B: Sciatica: what the rheumatologist needs to know. Nat Rev Rheumatol 6:139-145, 2010

28. Wang H, Huang B, Li C, Zhang Z, Wang J, Zheng W, et al: Learning curve for percutaneous endoscopic lumbar discectomy depending on the surgeon's training level of minimally invasive spine surgery. Clin Neurol Neurosurg 115:19871991, 2013

29. Wang H, Zhou Y, Li C, Liu J, Xiang L: Risk factors for failure of single-level percutaneous endoscopic lumbar discectomy. J Neurosurg Spine 23:320-325, 2015

\section{Disclosures}

The authors report no conflict of interest concerning the materials or methods used in this study or the findings specified in this paper.

\section{Author Contributions}

Conception and design: Harhangi, Gadjradj, Peul. Acquisition of data: Harhangi, Gadjradj. Analysis and interpretation of data: Harhangi, Gadjradj, Peul. Drafting the article: Harhangi, Gadjradj, van Tulder, Dirven. Critically revising the article: Harhangi, van Tulder, Dirven, Peul. Reviewed submitted version of manuscript: all authors. Statistical analysis: Harhangi, Gadjradj, Peul. Administrative/technical/material support: Harhangi, van Tulder. Study supervision: Harhangi, van Tulder, Dirven, Peul.

\section{Correspondence}

B. Sanjay Harhangi, Department of Neurosurgery, Erasmus MC, 's-Gravendijkwal 230, Rotterdam 3015 CE, The Netherlands. email: b.s.harhangi@erasmusmc.nl. 